\title{
Efecto del catéter de hemodiálisis en la permeabilidad de fístulas arteriovenosas
}

\author{
Gregory Córdova A. ${ }^{1,2}$, Gonzalo Sáez C. ${ }^{3}$, Óscar Chávez C. ${ }^{3}$ y Francisco Tapia P. ${ }^{3}$
}

\section{The impact of hemodialysis catheter on arteriovenous fistula permeability}

Objective: To evaluate the effects of hemodialysis catheter on arteriovenous fistula (AVF) permeability. Materials and Method: We conducted a retrospective cohort study, including 195 patients randomly selected from all patients diagnosed with chronic renal failure, who had their first arteriovenous fistula between January 2014 and December 2018. The outcomes were arteriovenous fistula thrombosis and permeability. For data analysis we used Chi-square test; Relative-Risk (CI 95\%); Kaplan-Meier analysis and Cox regression; p value less than 0.05 were considered as significant. Results: The $52.3 \%$ of the patients used hemodialysis catheter, from this group, the $49.5 \%$ had AVF thrombosis versus the $17.7 \%$ of the group without history of hemodialysis catheter $(p<0.001)$, estimating in the univariate analysis a risk of thrombosis 2.7 times higher in patients with catheter before the creation of their AVF (CI 95\% 1.7 a 4.4), as well as, in the multivariate analysis the risk of AVF thrombosis was 2.8 times higher in this group of patients, being identified the history of hemodialysis catheter as the only significative risk factor for thrombosis. Additionally, the AVF permeability time in this group was significantly less $(p<0.001)$ than patients without history of hemodialysis catheter (28.1 vs 43.9 months). Conclusion: We identified the hemodialysis catheter as a risk factor of arteriovenous fistula thrombosis, decreasing significantly its permeability time.

Key words: arteriovenous fistula; hemodialysis catheter; thrombosis.

\section{Resumen}

Objetivo: Evaluar el impacto que tiene el uso de catéteres de hemodiálisis en la permeabilidad de fístulas arteriovenosas. Materiales y Método: Estudio observacional, analítico, tipo cohorte retrospectiva. Se calculó un tamaño muestral de 195 pacientes, seleccionados aleatoriamente a partir del total de pacientes diagnosticados con enfermedad renal crónica (ERC) terminal, a quienes se les realizó su primera fístula arteriovenosa (FAV) entre enero de 2014 y diciembre de 2018. Como variables resultado se consideraron la trombosis de FAV y el tiempo de permeabilidad. Para el análisis inferencial se utilizaron las pruebas de Chi cuadrado; RR (IC 95\%); curvas Kaplan-Meier; regresión de Cox; considerando un valor de $\mathrm{p}$ significativo $<0,05$. Resultados: El 52,3\% utilizó catéter de hemodiálisis, de los cuales el 49,5\% presentó trombosis de su FAV versus el $17,7 \%$ del grupo sin este antecedente $(p<0,001)$; estimándose en el análisis univariado un riesgo de trombosis 2,7 veces mayor en pacientes con catéter previo a la confección de su FAV (IC $95 \% 1,7$ a 4,4). En el análisis multivariado, se identificó como único factor significativo el antecedente de catéter de hemodiálisis, estimándose que los pacientes usuarios de catéter previo a la confección de su FAV tienen 2,8 veces más riesgo de trombosis en el tiempo que quienes no utilizaron catéter (IC 95\% 1,6 a 4,9), quienes además presentaron un tiempo de permeabilidad significativamente menor $(p<0,001)$ en comparación con pacientes sin antecedente de catéter ( 28,1 vs 43,9 meses). Conclusión: Identificamos el uso del catéter de hemodiálisis como un factor de riesgo de trombosis de fístulas arteriovenosas, afectando significativamente su permeabilidad en el tiempo.

Palabras clave: fístula arteriovenosa; catéter de hemodiálisis; trombosis.
Hospital San Juan de Dios. Los Andes, Chile. ¿Universidad de Valparaíso. Valparaíso, Chile. Internos de Medicina Universidad de Valparaíso. Valparaíso, Chile.

Recibido 2019-08-27 y aceptado 2020-04-14

Correspondencia a: Dr. Gregory Córdova A gregory.cordova@uv.cl 


\section{Introducción}

La enfermedad renal crónica constituye uno de los principales problemas de salud en la actualidad, con una prevalencia estimada de un $10 \%$ a nivel mundial, donde la mayor parte de los pacientes desconocen su diagnóstico, presentándose habitualmente en etapas avanzadas ${ }^{1,2}$.

Según reportes internacionales, la prevalencia de enfermedad renal crónica terminal (ERCt) en adultos mayores de 65 años, aumentó de 4.156 por millón de habitantes en el año 2000 a 6.223 por millón de habitantes en el año $2015^{3}$, encontrándose el $45,9 \%$ de los pacientes de este grupo etario en etapa terminal ${ }^{4}$.

En nuestro país, la Encuesta Nacional de Salud de 2009-2010, reportó una prevalencia de enfermedad renal crónica de $2,7 \%$ en la población general, mientras que un estudio realizado en la ciudad de Concepción que incluyó 27.894 pacientes adultos pertenecientes a un centro de salud familiar estimó una prevalencia global de enfermedad renal crónica de un $12,1 \%$, donde el $14,5 \%$ correspondía a mujeres y el 23,3\% eran mayores de 60 años ${ }^{1}$.

De acuerdo con la guía clínica "Kidney Disease Outcomes Quality Initiative" (KDOQI), la fístula arteriovenosa (FAV) autóloga debe ser el acceso vascular de elección, debido a que ésta tiene un menor riesgo de complicaciones, un mayor tiempo de permeabilidad y una mayor tasa de sobrevida, en comparación a otros accesos vasculares ${ }^{5,6}$. En relación a esto, se llevó a cabo en Estados Unidos el año 2003, una iniciativa para mejorar la calidad de accesos vasculares de hemodiálisis llamada "Fistula First Initiative" (FFI), cuyo objetivo principal fue aumentar el uso de fístulas arteriovenosas autólogas en pacientes con enfermedad renal crónica terminal de $40 \%$ a $65 \%$ para el año 2009 . Si bien, la cantidad de pacientes con enfermedad renal crónica terminal que utilizan fístulas autólogas ha aumentado progresivamente en la última década gracias a esta propuesta, el principal objetivo asociado es mantener una adecuada funcionalidad y permeabilidad a través de los años?

Se ha visto en diferentes estudios que el uso de catéter venoso de hemodiálisis (CHD) en enfermedad renal crónica terminal aumenta el riesgo de infección, sepsis, mortalidad, disminuye la calidad de vida y reduce las tasas de flujo en comparación con los injertos protésicos y las fístulas autólogas, además de incrementar el riesgo de estenosis venosa central ${ }^{7}$. A pesar de las recomendaciones de guías clínicas nacionales y extranjeras, en EE.UU. el $80 \%$ de los pacientes que inician hemodiálisis lo hacen a través de un catéter venoso en lugar de una fístula arteriovenosa (autóloga o protésica) ${ }^{8}$, siendo una tendencia que se repite en algunos países como España y Francia, donde se ha triplicado y duplicado su uso respectivamente ${ }^{9}$.

Una revisión norteamericana multicéntrica ${ }^{8}$, incluyó a 476.926 pacientes que iniciaron hemodiálisis entre los años 2007 y 2011 , reportando que el $16 \%$ comenzó con fístula autóloga, el $3 \%$ con fístula protésica y el $81 \%$ con catéter venoso; de estos últimos, el $28 \%$ se convirtió a fístula autóloga y el $9 \%$ a protésica dentro del período de estudio, dejando al $63,8 \%$ de los pacientes en hemodiálisis por catéter. Según cifras nacionales del Ministerio de Salud, del total de pacientes ingresados a hemodiálisis al 31 de agosto del año 2018, el 21,1\% ingresó con una fístula arteriovenosa autóloga madura, el 6,3\% con una fístula arteriovenosa autóloga inmadura, el 57,2\% con catéter venoso de hemodiálisis permanente, el $14,2 \%$ con catéter transitorio y el $1,2 \%$ con fístula arteriovenosa protésica ${ }^{10}$.

Considerando que los problemas con los accesos vasculares son la principal causa de hospitalización de pacientes con enfermedad renal crónica terminal, y que más del $60 \%$ de ellos tuvo un catéter venoso previo a la confección de un acceso vascular definitivo $^{10}$, este estudio tiene como objetivo principal evaluar el efecto que tiene el uso de catéteres de hemodiálisis en la permeabilidad de fístulas arteriovenosas a corto y mediano plazo identificando la asociación entre el uso de catéter y trombosis de la FAV. Además, nuestros objetivos secundarios fueron estimar el riesgo de trombosis de la FAV en pacientes usuarios de catéter de hemodiálisis preoperatorio, y comparar la permeabilidad promedio de la FAV entre pacientes con y sin antecedente de catéter.

\section{Materiales y Método}

\section{Tipo de estudio}

Se realizó un estudio observacional, analítico, tipo cohorte retrospectiva, con el fin de poder evaluar y comparar resultados entre pacientes operados en distintas fechas y con diferentes períodos de seguimiento, pudiendo de este modo, observar con mayor claridad el impacto en el tiempo del uso de catéteres de hemodiálisis en la permeabilidad de fístulas arteriovenosas.

\section{Universo y muestra}

El universo corresponde a todos los pacientes del Hospital de Los Andes con diagnóstico de enfermedad renal crónica terminal a quienes se les realizó 
su primera fístula arteriovenosa entre enero de 2014 y diciembre de 2018 con seguimiento hasta mayo de 2019, calculándose un tamaño muestral de 195 pacientes mediante el programa $G^{*}$ Power, con una potencia estadística del $80 \%$ y un nivel de confianza de $95 \%$, los cuales fueron seleccionados de manera aleatoria a partir de una base de datos del servicio de cirugía del hospital. Los pacientes operados fueron evaluados a las 3 y 6 semanas y, posteriormente, a los 6 y 12 meses, recibiendo atención fuera de estas fechas aquellos pacientes derivados directamente desde los centros de diálisis que presentaron algún tipo de complicación de su fístula arteriovenosa. Para aquellos pacientes que no reportaron complicaciones dentro de los primeros 12 meses ni posterior a este periodo hasta la fecha de término de este estudio, se contactó directamente a los centros de diálisis y/o mediante el registro clínico electrónico para confirmar la adecuada permeabilidad y funcionamiento de su acceso vascular.

\section{Base de datos}

Se creó una base de datos en el programa Microsoft Excel versión 16.25, donde se registraron las siguientes variables aleatorias: fecha de nacimiento, edad, sexo, comorbilidades (diabetes mellitus; hipertensión arterial; insuficiencia cardiaca; patología respiratoria crónica; dislipidemia; patología tiroidea), hábitos (tabaquismo; consumo de alcohol; consumo de drogas), uso catéter de hemodiálisis previo a creación de acceso definitivo (catéter transitorio o tunelizado), fecha de creación y tipo de fístula arteriovenosa (autóloga; protésica), presencia de trombosis de fístula arteriovenosa (definida como ausencia de flujo por presencia de material trombótico en el trayecto de la fístula arteriovenosa), y meses de permeabilidad desde su creación. Los datos fueron recolectados directamente desde los pacientes y sus registros clínicos en cada control médico, antes y después de la confección de la fístula arteriovenosa e ingresados a la base de datos general desde donde se rescató la información utilizada para este estudio, contactando directamente en los centros de diálisis y/o revisando los registros clínicos electrónicos para obtener datos faltantes.

\section{Análisis estadístico}

Para el análisis se utilizó el software estadístico SPSS versión 25 , las variables categóricas se describen con porcentajes y número de casos, mientras que las variables cuantitativas con medias y desviación estándar. En el análisis inferencial se utilizó la prueba Chi cuadrado para evaluar asociación entre variables; como estimador de riesgo se calcularon riesgos relativos con sus intervalos de confianza al 95\%; el análisis de supervivencia se realizó mediante curvas de Kaplan-Meier evaluando diferencias con LogRank Test; y para el análisis multivariado se utilizó la regresión de Cox; se consideró en todas las pruebas estadísticas un valor de $\mathrm{p}$ significativo menor de 0,05 .

\section{Control de sesgos}

Para controlar sesgos de selección, los pacientes incluidos en este estudio fueron seleccionados aleatoriamente a partir de una base de datos que incluía a todos los pacientes con diagnóstico de enfermedad renal crónica terminal en hemodiálisis a quienes se les realizó su primera fístula arteriovenosa en nuestro hospital. Para controlar sesgos de medición, se utilizaron registros clínicos para confirmar la veracidad de la información entregada por los pacientes seleccionados, mientras que los sesgos de confusión fueron controlados mediante la aplicación de análisis estadístico multivariado.

\section{Resultados}

\section{Caracterización de la muestra}

De los 195 pacientes seleccionados aleatoriamente todos cumplían con los criterios de inclusión, pudiendo obtenerse la información completa de las variables incluidas en este estudio desde la base de datos del servicio en 154 casos, debiendo rescatarse dirigidamente datos incompletos de variables de los 41 pacientes restantes, desde los registros clínicos físicos y/o electrónicos, así como también, mediante contacto directo con los centros de diálisis, lográndose un adecuado seguimiento retrospectivo en el total de la muestra utilizada. De los 195 pacientes portadores de ERC en hemodiálisis, 96 de ellos fueron de sexo masculino $(49,2 \%)$ y 99 de sexo femenino $(50,8 \%)$, con una media de edad de 61,5 años, y un promedio de permeabilidad global de las FAV de 15,9 meses (Tabla 1a). Dentro de las comorbilidades más frecuentes se encontró la HTA (153 pacientes, 78,5\%) y la diabetes mellitus (110 pacientes, 56,4\%), seguidas por la insuficiencia cardiaca crónica (29 pacientes, 14,9\%), la patología tiroidea (25 pacientes, $12,8 \%$ ) y la dislipidemia (19 pacientes, 9,7\%), observándose en un pequeño porcentaje la presencia de patologías respiratorias crónicas ( 9 pacientes, 4,6\%) (Tabla 1b).

\section{Uso de catéter de hemodiálisis, tipo de fístula arteriovenosa y trombosis}

Del total de la muestra, 99 pacientes $(50,8 \%)$ utilizaron un catéter venoso de hemodiálisis antes de la 
Tabla 1a. Caracterización de la muestra. Variables cuantitativas

\begin{tabular}{|lccc|}
\hline Variables numéricas & Media (DE) & Mínimo-máximo & IC 95\% \\
\hline Edad (años) & $61,5(14,8)$ & 20 a 80 & 59,4 a 63,6 \\
\hline Permeabilidad (meses) & $15,9(11,1)$ & 1 a 59 & 14,3 a 17,4 \\
\hline
\end{tabular}

Tabla 1b. Caracterización de la muestra. Variables categóricas

\begin{tabular}{|lrc|}
\hline Variables categóricas & n $(\%)$ & IC $\mathbf{9 5 \%}$ \\
$\begin{array}{l}\text { Sexo } \\
\text { • Masculino }\end{array}$ & $96(49,2)$ & 42,2 a 56,2 \\
- Femenino & $99(50,8)$ & 43,7 a 57,8 \\
DM & $110(56,4)$ & 0,49 a 0,63 \\
HTA & $153(78,5)$ & 72,7 a 84,2 \\
ICC & $29(14,9)$ & 9,9 a 19,9 \\
Dislipidemia & $19(9,7)$ & 5,5 a 13,8 \\
Pat. respiratoria & $9(4,6)$ & 1,6 a 7,5 \\
Pat. tiroidea & $25(12,8)$ & 8,1 a 17,4 \\
Tabaquismo & $32(16,4)$ & 11,2 a 21,6 \\
OH & $17(8,7)$ & 4,7 a 12,6 \\
\hline
\end{tabular}

Tabla 2. Uso de catéter de hemodiálisis, tipo de fístulas arteriovenosas y porcentaje de trombosis

\begin{tabular}{|lrc|}
\hline Variables categóricas & $\mathbf{n}(\mathbf{\%})$ & IC 95\% \\
\hline Uso catéter preoperatorio & & \\
- Sí & $102(52,3)$ & 45,2 a 59,3 \\
- No & $93(47,7)$ & 40,6 a 54,7 \\
Tipo de fístula arteriovenosa & & \\
- Autóloga & $185(94,9)$ & 91,8 a 97,9 \\
- Protésica & $6(3,1)$ & 0,6 a 5,5 \\
- Super. Basílica & $4(2,1)$ & 0,09 a 4,1 \\
Trombosis de fístula arteriovenosa & & \\
- Sí & $66(33,8)$ & 27,1 a 40,4 \\
- No & $129(66,2)$ & 59,5 a 72,8 \\
\hline
\end{tabular}

Tabla 3. Trombosis de fístulas arteriovenosas según uso de catéter de hemodiálisis

\begin{tabular}{|lccc|}
\hline & \multicolumn{4}{c|}{ Trombosis de fístulas arteriovenosas n (\%) } \\
Uso de catéter preop. & Sí & No & Total \\
- Sí & $49(49,5)$ & $50(50,5)$ & $99(100)$ \\
- No & $17(17,7)$ & $79(82,3)$ & $96(100)$ \\
& $66(100)$ & $129(100)$ & $195(100)$ \\
\hline
\end{tabular}

$\chi^{2} 19.242$ valor $\mathrm{p}<0,001$. confección de su fístula arteriovenosa. En relación a los tipos de fístulas creadas, a 185 pacientes $(94,9 \%)$ se les realizó una fístula arteriovenosa autóloga, a 6 pacientes $(3,1 \%)$ una fístula protésica y a 4 pacientes $(2,1 \%)$ una superficialización de vena basílica. Durante el periodo de estudio, 66 pacientes $(33,8 \%)$ presentaron trombosis de su fístula arteriovenosa (Tabla 2).

\section{Asociación entre uso de catéter de hemodiálisis y trombosis de FAV}

De los 99 pacientes que utilizaron catéter de hemodiálisis antes de la confección de su fístula arteriovenosa, 49 de ellos (49,5\%) presentaron trombosis de su fístula durante el periodo de estudio, cifra significativamente mayor que la observada en el grupo de pacientes sin catéter previo, donde se registró sólo en $17(17,7 \%)$ casos (valor $\mathrm{p}<0,001)$. Se calculó, que los pacientes que utilizan un catéter venoso de hemodiálisis previo a la creación de su FAV tienen un riesgo de trombosis 2,7 veces mayor (IC 95\% 1,7 a 4,4) que aquellos sin este antecedente (Tabla 3 y Figura 1). Por otro lado, en el análisis bivariado no se encontraron asociaciones significativas entre trombosis y el resto de las variables estudiadas (Tabla 4).

\section{Meses de permeabilidad de la FAV según uso de catéter de hemodiálisis}

En el análisis de supervivencia, pudimos observar que el grupo de pacientes con antecedente de catéter de hemodiálisis presentaron un tiempo de permeabilidad de su FAV significativamente menor ( $\mathrm{p}<0,001)$, correspondiente a 28,1 meses, comparado con el grupo de pacientes sin este antecedente, cuya permeabilidad fue de 43,9 meses (Tabla 5). Destaca, además, que el $50 \%$ de los pacientes con antecedente de catéter venoso tenían su fístula arteriovenosa trombosada a los 23 meses desde la creación del acceso vascular. Como se puede observar en las curvas de Kaplan-Meier, hay una clara diferencia en la permeabilidad entre ambos grupos a los 12, 24 y 36 meses de seguimiento (Figura 2).

\section{Análisis multivariado}

Al considerar todas las variables registradas en este estudio y su asociación con la trombosis del acceso vascular, se identificó el uso del catéter de hemodiálisis preoperatorio como el único factor de riesgo de trombosis de la fístula arteriovenosa a corto y mediano plazo, estimándose que los pacientes usuarios de catéter previo a la confección de su FAV, tienen 2,8 veces más riesgo de trombosis en el tiempo que quienes no utilizaron catéter (IC 95\% 1,6 a 4,9) (Tabla 6). 
Tabla 4. Asociación entre variables categóricas y trombosis de fístulas arteriovenosas

\begin{tabular}{|c|c|c|}
\hline Variable & $\begin{array}{c}\text { n }(\%) \\
\text { trombosis }\end{array}$ & Valor de $p$ \\
\hline $\begin{array}{l}\text { Sexo } \\
\text { - Masculino } \\
\text { - Femenino }\end{array}$ & $\begin{array}{l}29(43,9) \\
37(56,1)\end{array}$ & 0,290 \\
\hline $\begin{array}{l}\text { Diabetes mellitus } \\
\text { - Sí } \\
\text { - No }\end{array}$ & $\begin{array}{l}35(53) \\
31(47)\end{array}$ & 0,496 \\
\hline $\begin{array}{l}\text { Hipertensión arterial } \\
\text { - Sí } \\
\text { - No }\end{array}$ & $\begin{array}{l}49(74,2) \\
17(25,8)\end{array}$ & 0,305 \\
\hline $\begin{array}{l}\text { Dislipidemia } \\
\text { - Sí } \\
\text { - No }\end{array}$ & $\begin{array}{r}6(9,1) \\
60(90,9)\end{array}$ & 0,826 \\
\hline $\begin{array}{l}\text { ICC } \\
\text { - Sí } \\
\text { - } \mathrm{No}\end{array}$ & $\begin{array}{l}13(19,7) \\
53(80,3)\end{array}$ & 0,176 \\
\hline $\begin{array}{l}\text { Pat. respiratoria } \\
\text { - Sí } \\
\text { - No }\end{array}$ & $\begin{array}{r}4(6,1) \\
62(93,9)\end{array}$ & 0,491 \\
\hline $\begin{array}{l}\text { Pat. tiroidea } \\
\text { - Sí } \\
\text { - No }\end{array}$ & $\begin{array}{r}5(7,6) \\
61(92,4)\end{array}$ & 0,117 \\
\hline $\begin{array}{l}\text { Tabaquismo } \\
\text { - Sí } \\
\text { - No }\end{array}$ & $\begin{array}{l}13(19,7) \\
53(80,3)\end{array}$ & 0,375 \\
\hline $\begin{array}{c}\text { OH } \\
\text { - Sí } \\
\text { - } \mathrm{No}\end{array}$ & $\begin{array}{l}7(10,6) \\
59(89,4)\end{array}$ & 0,504 \\
\hline $\begin{array}{l}\text { Tipo de FAV } \\
\text { - Autóloga } \\
\text { - } \text { Protésica } \\
\text { - } \quad \text { Super. Basílica }\end{array}$ & $\begin{array}{rr}61 & (92,4) \\
4 & (6,1) \\
1 & (1,5)\end{array}$ & 0,213 \\
\hline
\end{tabular}

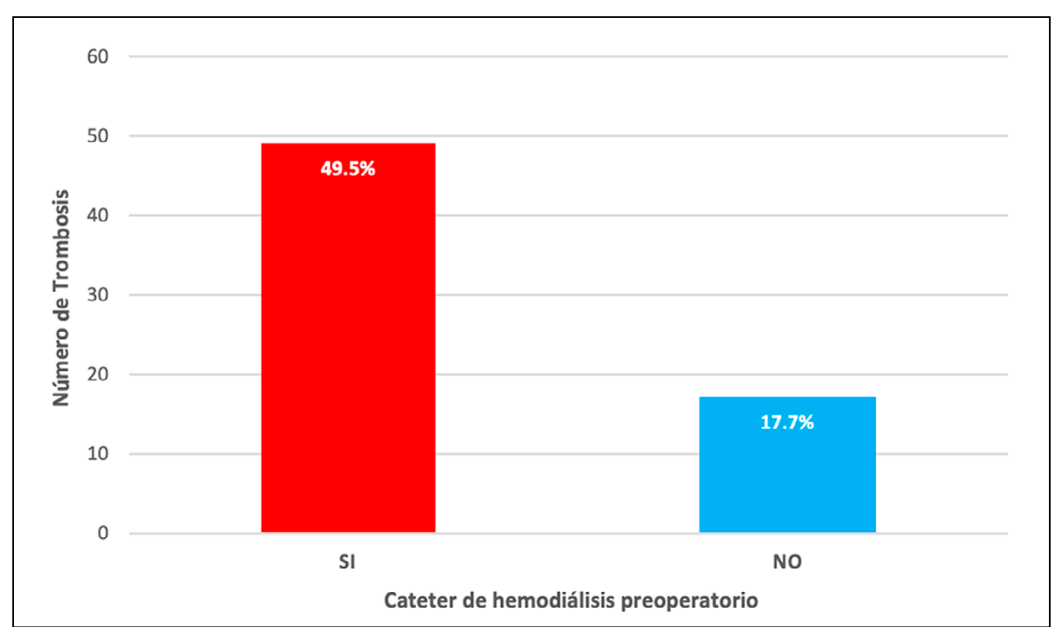

Figura 1. Trombosis de FAV según uso de catéter de hemodiálisis. Del grupo de pacientes con catéter de hemodiálisis preoperatorio, 49 de ellos (49,5\%) presentaron trombosis de su fístula arteriovenosa durante el periodo de estudio, cifra significativamente mayor que la observada en el grupo de pacientes sin este antecedente, donde se registró sólo en $17(17,7 \%)$ casos (valor $p<0,001)$.

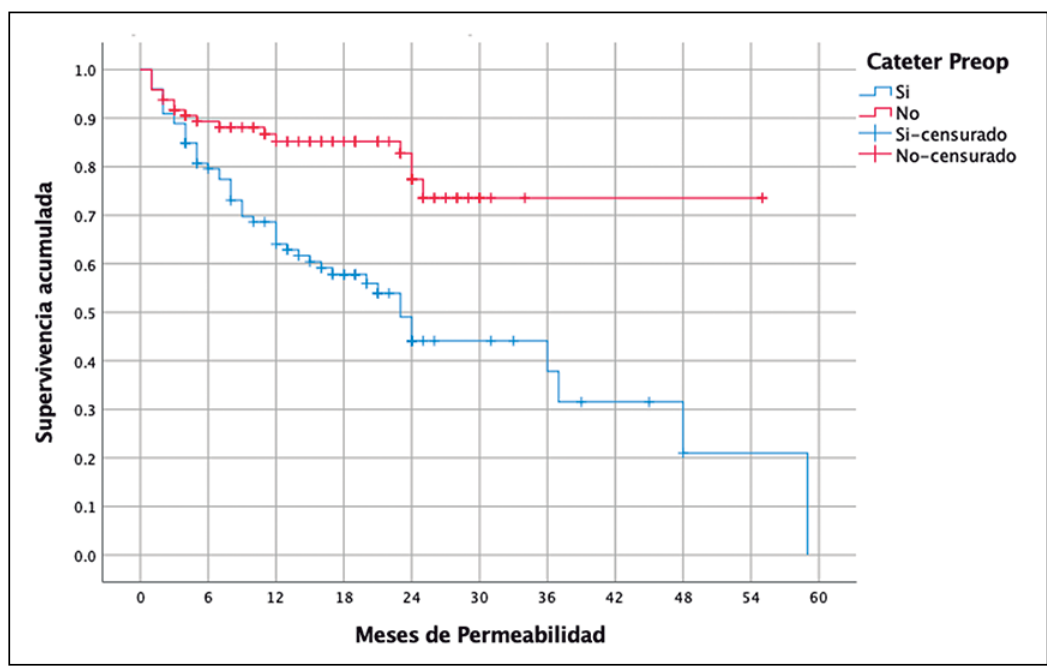

Figura 2. Permeabilidad de FAV según uso de catéter de hemodiálisis. Como se puede observar en las curvas de Kaplan-Meier, hay una clara diferencia en la permeabilidad entre ambos grupos a los 12, 24 y 36 meses de seguimiento (Log Rank test $p<0,001$ ). Los datos censurados corresponden a los pacientes que presentaban su fístula permeable al momento de la evaluación posoperatoria.

Tabla 5. Meses de permeabilidad de fístulas arteriovenosas según uso de catéter de hemodiálisis

\begin{tabular}{|lcccc|}
\hline Catéter preoperatorio & Media (DE) & IC 95\% & Mediana (DE) & IC 95\% \\
\hline - Sí & $28,1(2,9)$ & 22,4 a 33,8 & $23(2)$ & 18,9 a 27 \\
- No & $43,9(2,4)$ & 39,1 a 48,7 & & \\
- Total & $35(2,5)$ & 30 a 40 & $37(8,2)$ & 20,8 a 53,1 \\
\hline
\end{tabular}

Log Rank Test $\left(\chi^{2} 14,1\right)$ valor de $\mathrm{p}<0,001$. 
Tabla 6. Análisis Multivariado (Regresión Cox)

\begin{tabular}{|lcc|}
\hline Variable & HR & IC 95\% \\
\hline Catéter venoso preoperatorio & 2,8 & 1,6 a 4,9 \\
\hline
\end{tabular}

\section{Discusión}

El uso de catéteres de hemodiálisis es una práctica habitual en pacientes con enfermedad renal crónica terminal que requieren iniciar hemodiálisis y aún no cuentan con un acceso vascular definitivo que permita llevar a cabo este proceso de una manera segura, con una menor tasa de complicaciones y otorgando una mejor calidad de vida.

De los 195 pacientes estudiados, 102 de ellos, correspondientes al 52,3\% (IC 95\% 45,2 a 59,3), tienen antecedente de cateterismo yugular o subclavio previo a la confección de una fístula arteriovenosa, cifra significativamente menor a la reportada en estudios nacionales e internacionales, donde su uso fue observado hasta en el $70 \%$ de los casos. Por otro lado, destaca el alto porcentaje de pacientes que iniciaron su hemodiálisis a través de una fístula arteriovenosa autóloga, superando el $95 \%$ de los casos, siendo un número significativamente mayor al mencionado en estudios previos, donde no superaron el $27 \%$ del total ${ }^{8,10}$.

Nuestro estudio identificó el uso del catéter de hemodiálisis previo a la confección de la FAV como un factor de riesgo de trombosis del acceso vascular definitivo, observándose en el $49,5 \%$ de los pacientes que utilizaron previamente catéter, y sólo en el $17,7 \%$ de aquellos sin este antecedente (valor $\mathrm{p}<0,001$ ). Al incluir la variable tiempo, encontramos que los pacientes usuarios de catéter de hemodiálisis, tuvieron un tiempo de permeabilidad significativamente menor (valor $\mathrm{p}<0,001$ ) en comparación con quienes no tuvieron catéter antes de la creación de su FAV, correspondiente a 28,1 y 43,9 meses respectivamente; estimándose además, que los pacientes con cateterismo venoso previo tienen entre 1,6 a 4,9 veces más riesgo de trombosis durante su seguimiento que aquellos sin uso de catéter preoperatorio (HR 2,8/IC 95\% 1,6 a 4,9). A diferencia de otras publicaciones, en el análisis multivariado no encontramos asociación de trombosis con otras variables como edad y factores metabólicos ${ }^{11,12}$.

Estos resultados, sugieren que uso de catéteres venosos centrales previos a la confección y durante la maduración de las fístulas arteriovenosas (autólogas o protésicas) interfieren de alguna manera con el retorno venoso, alterando el perfil hemodinámico y favoreciendo el desarrollo de trombosis. Dentro de las posibles causas, múltiples estudios han asociado el uso de catéteres venosos centrales como catéteres de hemodiálisis o sondas de marcapasos con el desarrollo de estenosis venosa central ${ }^{5,11}$, lo que podría justificar los resultados observados. Sin embargo, el número de pacientes que presentan clínica relevante de estenosis venosa central como edema facial, mamario o de extremidades, o circulación colateral son limitados, como ha sido demostrado en un estudio donde se evaluó retrospectivamente a 2.856 pacientes en hemodiálisis con acceso vascular fallido donde sólo 26 pacientes tuvieron síntomas evidentes de estenosis venosa central ${ }^{13}$. Destaca además, que 19 de ellos, no presentaron antecedente de cateterización yugular o subclavia, planteando una nueva hipótesis ya respaldada por estudios previos donde la estenosis venosa central no depende exclusivamente de la injuria mecánica del cateterismo crónico, sino también puede responder a alteraciones hemodinámicas que generan estrés parietal, turbulencia y agregación plaquetaria, así como también, compresión extrínseca de venas centrales por estructuras mediastínicas, poniendo en duda un concepto ampliamente aceptado del efecto mecánico del cateterismo como causa predominante de estenosis venosa central ${ }^{14-19}$.

Por otro lado, considerando lo expuesto previamente, se podría presumir que un porcentaje no menor de pacientes en hemodiálisis podría presentar estenosis venosa central asintomática, y que su tratamiento endovascular profiláctico podría prevenir el fracaso de la fístula arteriovenosa en estos pacientes, sin embargo, estudios han demostrado que el tratamiento de estenosis venosas mayores al 50\% asintomáticas ha sido asociado con mayor progresión en el grado de estenosis y empeoramiento de las lesiones, en comparación con pacientes tratados conservadoramente ${ }^{20}$.

Múltiples estudios han demostrado que el mapeo vascular preoperatorio aumenta significativamente la tasa de éxito y permeabilidad de las fístulas arteriovenosas autólogas, sugiriendo un tamaño intraluminal venoso mínimo entre 2,0 a $2,5 \mathrm{~mm}$ y arterial mayor a $1,6 \mathrm{~mm}$ para asegurar una adecuada maduración y permeabilidad ${ }^{21-23}$, sin embargo, a pesar de que en esta serie a todos los pacientes se les realizó una evaluación ecográfica preoperatoria para elegir el sitio del acceso vascular, la falta de rigurosidad en el registro sobre el tamaño y la calidad de los vasos seleccionados, impidió incluir esta variable en nuestro estudio, siendo un potencial sesgo de confusión para evaluar el real impacto de 
nuestra variable exposición (el uso de catéter de hemodiálisis previo a la creación de una FAV) sobre la permeabilidad de las fístulas arteriovenosas, por lo que se vuelve fundamental considerarla en estudios prospectivos posteriores. Consideramos que, a pesar del potencial sesgo mencionado anteriormente, los resultados obtenidos muestran con claridad una importante asociación entre el uso de catéter de hemodiálisis y el desarrollo precoz de trombosis de fístulas arteriovenosas, asociación visualizada comúnmente en la práctica clínica y respaldada con los hallazgos de este estudio, pudiendo generalizarse nuestros resultados criteriosamente a poblaciones similares.

\section{Conclusión}

Nuestro estudio identificó el uso del catéter de hemodiálisis como un factor de riesgo de trombosis de fístulas arteriovenosas a corto y mediano plazo, afectando significativamente su permeabilidad en el tiempo. Aunque el desarrollo de estenosis venosa central secundario al cateterismo yugular o subclavio previo podría corresponder a la causa más probable, existe evidencia contradictoria en relación a su verdadera etiología y manejo, sobre todo en pacientes asintomáticos. Se vuelve indispensable la realización de investigaciones de carácter prospectivo para reducir los sesgos potenciales encontrados en nuestro estudio relacionados principalmente con la exactitud del registro y seguimiento. De este modo, podremos obtener un mayor entendimiento de esta patología que nos permita definir estrategias efectivas para un manejo oportuno.

\section{Responsabilidades éticas}

Protección de personas y animales. Los autores declaran que para esta investigación no se han realizado experimentos en seres humanos ni en animales.

Confidencialidad de los datos. Los autores declaran que en este artículo no aparecen datos de pacientes.

Financiamiento: No se requirió apoyo financiero ni patrocinio para la realización de este estudio.

Conflictos de interés: no hay.

\section{Bibliografía}

1. Zúñiga SMC, Muller OH, Flores OM Prevalence of chronic kidney disease in subjects consulting in urban primary care clinics. Rev Med Chile 2011;139:1176-84.

2. Vassalotti JA, Centor R, Turner BJ, Greer RC, Choi M, Sequist TD, et al. Practical Approach to Detection and Management of Chronic Kidney Disease for the Primary Care Clinician. Am J Med. 2016;129:153-62e7.

3. Collins AJ, Foley RN, Chavers B, Gilbertson D, Herzog C, Johansen K, et al. United States Renal Data System 2011 Annual Data Report: Atlas of chronic kidney disease $\&$ end-stage renal disease in the United States. American journal of kidney diseases: the official journal of the National Kidney Foundation 2012;59(1 Suppl 1):A7,e1-420.

4. Jin DC, Yun SR, Lee SW, Han SW, Kim W, Park J, et al. Current characteristics of dialysis therapy in Korea: 2016 registry data focusing on diabetic patients. Kidney Res Clin Pract. 2018;37:20-9.

5. National Kidney F. KDOQI Clinical Practice Guideline for Hemodialysis Adequacy: 2015 update. American journal of kidney diseases: the official journal of the National Kidney Foundation 2015;66:884-930.

6. Bae E, Lee H, Kim DK, Oh KH, Kim YS, Ahn $\mathrm{C}$, et al. Autologous arteriovenous fistula is associated with superior outcomes in elderly hemodialysis patients. BMC Nephrol. 2018;19:306.

7. Lok CE. Fistula first initiative: advantages and pitfalls. Clin J Am Soc Nephrol. 2007;2:1043-53.

8. Arhuidese IJ, Orandi BJ, Nejim B, Malas M. Utilization, patency, and complications associated with vascular access for hemodialysis in the United States. J Vasc Surg. 2018;68:1166-74.

9. Ethier J, Mendelssohn DC, Elder SJ, Hasegawa T, Akizawa T, Akiba T, et al. Vascular access use and outcomes: an international perspective from the Dialysis Outcomes and Practice Patterns Study.
Nephrol Dial Transplant. 2008;23:321926.

10. Poblete H. XXXVIII Cuenta de hemodiálisis crónica en Chile. Sociedad Chilena de Nefrología, Registro de Diálisis 2018;38:27-9.

11. Ocak G, Rotmans JI, Vossen CY, Rosendaal FR, Krediet RT, Boeschoten $\mathrm{EW}$, et al. Type of arteriovenous vascular access and association with patency and mortality. BMC Nephrol. 2013;14:79.

12. Cheng Q, Zhao YJ. The reasons for the failure of the primary arteriovenous fistula surgery in patients with end-stage renal disease. The journal of vascular access. 2015;16 Suppl 10:S74-7.

13. Kotoda A, Akimoto T, Kato M, Kanazawa H, Nakata M, Sugase T, et al. Central venous stenosis among hemodialysis patients is often not associated with previous central venous catheters. ASAIO J. 2011;57:439-43.

14. Ashizawa A, Kimura G, Sanai T, Inenaga $\mathrm{T}$, Kawano $\mathrm{Y}$, Matsuoka $\mathrm{H}$, et al. Idiopathic left innominate vein stenosis manifested following the creation of 


\section{ARTÍCULO ORIGINAL}

arteriovenous fistula in uremia. Am J Nephrol. 1994;14:142-4.

15. Williams ME. Venous thoracic outlet syndrome simulating subclavian stenosis in a hemodialysis patient. Am J Nephrol. 1998;18:562-4.

16. Morosetti M, Meloni C, Gandini R, Galderisi C, Pampana E, Nicoletti M, et al. Late symptomatic venous stenosis in three hemodialysis patients without previous central venous catheters. Artif Organs. 2000;24:929-31.

17. Kim H, Chung JW, Park JH, Yin YH, Park SH, Yoon CJ, et al. Role of CT venography in the diagnosis and treatment of benign thoracic central venous obstruction. Korean J Radiol.
2003;4:146-52.

18. Itkin M, Kraus MJ, Trerotola SO. Extrinsic compression of the left innominate vein in hemodialysis patients. J Vasc Interv Radiol. 2004;15(1 Pt 1):516.

19. Oguzkurt L, Tercan F, Yildirim S, Torun D. Central venous stenosis in haemodialysis patients without a previous history of catheter placement. Eur J Radiol. 2005;55:237-42.

20. Levit RD, Cohen RM, Kwak A, ShlanskyGoldberg RD, Clark TW, Patel AA, et al. Asymptomatic central venous stenosis in hemodialysis patients. Radiology. 2006;238:1051-6.

21. Silva MB Jr, Hobson RW 2nd, Pappas PJ,
Jamil Z, Araki CT, Goldberg MC, et al. A strategy for increasing use of autogenous hemodialysis access procedures: impact of preoperative noninvasive evaluation. J Vasc Surg. 1998;27:302-7; discussion 7-8.

22. Allon M, Lockhart ME, Lilly RZ,

Gallichio MH, Young CJ, Barker J, et al. Effect of preoperative sonographic mapping on vascular access outcomes in hemodialysis patients. Kidney Int. 2001;60:2013-20.

23. Mendes RR, Farber MA, Marston WA, Dinwiddie LC, Keagy BA, Burnham SJ. Prediction of wrist arteriovenous fistula maturation with preoperative vein mapping with ultrasonography. J Vasc Surg. 2002;36:460-3. 